\title{
A multi-period optimization model for the design of new LNG supply chains
}

\author{
Alice Bittante Henrik Saxén Frank Pettersson \\ Åbo Akademi University, Turku, Finland, \{alice.bittante, henrik.saxen, \\ frank.pettersson\} dabo.fi
}

\begin{abstract}
This paper presents a mathematical model developed to aid decision making in the design of a supply chain for liquefied natural gas (LNG). The supply problem considers the delivery of LNG from a number of supply ports to a set of consumers by maritime transportation to satellite terminals and by land-based truck transports from the terminals to consumers on or off the coast. The model addresses both tactical and strategic aspects in the design of a new supply chain, optimizing maritime routing of a heterogeneous fleet, truck connections and the strategic locations of the satellite terminals. The objective is to minimize the overall cost for the selected time horizon, considering both operation and investment costs. By contrast to an earlier effort by the authors, the present work also addresses storage sizes and inventory at the satellite terminals by applying a multi-period formulation. The performance of the model is illustrated by a case study, where the optimal LNG supply chain for a coastal region at a gulf was designed. The model was found to be a flexible tool for an initial design and feasibility analysis of small-scale LNG supply chains.
\end{abstract}

Keywords: Energy Systems; MILP; Optimization; Small Scale LNG; Supply Chain

\section{Introduction}

Liquefied natural gas (LNG) is produced by cooling natural gas (NG) below $-162{ }^{\circ} \mathrm{C}$ (at atmospheric pressure), which reduces the volume to approximately one six-hundredth of the original. It is the cleanest form of fossil fuels since it has undergone purification during the liquefaction process, and it has a high $\mathrm{H} / \mathrm{C}$ ratio as it is primarily composed of methane. The lower carbon intensity is the key of its recent growth of popularity: LNG is a perfect bridge fuel on the way to a world using $100 \%$ renewable energy, providing grid stability and reliability during fluctuations in the electricity generation of intermittent renewable sources. Furthermore, the recent regulations against pollution in the maritime transportation within identified emission control areas imposed by the International Maritime Organization (IMO) have also increased the popularity of LNG, making it the most suitable fuel for maritime vessels propulsion.
The rising interest in LNG and the lack of infrastructures have served to activate several LNGrelated projects worldwide. Among these, a new market segment of small-scale LNG supply chains has become increasingly important, promoting LNG utilization for middle- to small-scale applications and in sparsely distributed areas. Traditionally, the LNG supply chains have been designed for large volumes transported over long distances with LNG cargo capacities between $125,000 \mathrm{~m}^{3}$ and $140,000 \mathrm{~m}^{3}$ (but with tanker sizes up to $265,000 \mathrm{~m}^{3}$ currently available), in what is called liner shipping networks. Small-scale supply chains present specific characteristics and features, which differ substantially from larger chains. Vessels capacities vary from some thousand cubic meters to $30,000-40,000 \mathrm{~m}^{3}$ and the ship loads can be split on consecutive receiving ports. Demands are small and distributed over short distances, from hundred to a few thousand kilometers. Satellite terminals are equipped with small storage tanks $\left(<50,000 \mathrm{~m}^{3}\right)$ to be refilled once or a few times a month. Given the high investment cost involved in both infrastructure and operation of such supply networks, the optimization of the supply chain design can have a very significant effect on the overall economics of an LNG project.

The model presented in this paper is partially based on previous work by the authors (Bittante et al. 2016), where a single-period model was introduced to aid decision making on crucial aspects in the design of small-scale LNG supply chains. The work focused on both strategic and tactic decisions, simultaneously optimizing location of the satellite terminals, size of the fleet and vehicle routing to solve the overall logistic problem. The contribution of the current paper lies in the consideration of storage size and inventory level optimization, achieved through the adoption of a multiperiod formulation.

In general, the problem studied can be considered part of the broad research area of vehicle routing problems (VRP), for which the literature is rich and many variants and classes have been established (Braekers et al. 2015). However, classical VRP problems do not consider all aspects tackled by the proposed model. The subproblem defining the number and size of vessels needed to solve the maritime 
transportation represents a fleet-size and mix-vehicle routing problem (FSMVRP), while the strategic subproblem of locating the satellite terminals and the linked routing belongs to the class of location-routing problems (LRP). Finally, the sizing of storage tanks and the associated inventory include aspects attributable to the class of inventory routing problems (IRP). For more detailed information on these classes of problems we refer to Hoff et al. (2010) for a review paper on FSMVRP with both maritime and land transport, Drexl and Schneider (2015) for a recent survey and classification on LRP, and Andersson et al. (2010) for a comprehensive review on IRP.

Most of the approaches proposed in the literature focus on heuristics solutions and very few papers present exact methods. Baldacci et al. (2009) designed a mixed integer linear programming (MILP) model to solve a FSMVRP problem similar to the one considered in this paper, but where each customer is associated with a single route as in the classic VRP. By contrast, in our approach we allow for multiple visits at the customers by multiple vehicles. This feature also occurs in the formulation of the LRP subproblem. While most of the variables used for describing the activation of satellite terminal and their associated routes and delivery are the same as those used in the literature (Gonzalez-Feliu 2009), the set of binary variables to activate the routes associated with an activated satellite terminal has been changed into integer variables to allow for multiple voyages to the same port. According to the classification given in the review paper by Andersson et al. (2010), our approach falls into the finite time class, featuring deterministic demand, many-to-many topology, multiple routing, fixed inventory, and heterogeneous fleet composition. A contribution listed in the reviewed literature presenting similar features and where a deterministic approach is used is the work by AlKhayyal and Hwang (2007) for an IRP for a multicommodity liquid bulk. Their problem is a classic IRP problem, where storage sizes are given as well as the fleet of ships. The model focuses on the key aspects of a multi-product pickup-delivery problem and the authors solve a small illustrative example for detecting the central parameters to be used in developing a strategy for solving larger instances. A recent paper by Koza et al. (2017) presents many characteristics similar to our problem, including the focus on LNG market and infrastructure. Our contribution differs by the marketsegment it is directed to (i.e., small-scale logistics versus liner networks), the feature of locating the satellite terminals and the multi-period formulation for optimized storage sizing and inventory. Furthermore, our approach is based on an arc-flow formulation while the above-mentioned reference applies a path-based model.

The literature on routing problems often favors pathflow formulation over arc-based ones: with the feasible routes being predefined, the path-flow formulations do not include constraints related to routing feasibility, but focus on the set of ports or customers to serve. This is usually beneficial when the size of the problem increases. Often, the solution time is anyhow unacceptably long and pure heuristics or early terminations in exact methods are preferred. This is mainly due to the intrinsic complexity of VRP in general. Usually only very small problems, not relevant for real-life studies, can be solved in reasonable computation time with a deterministic approach. To overcome this limitation — still applying an exact method - we neglected some operational aspects that were not considered essential at the first level of planning: We do not consider scheduling, supply availability according to production rate or inventory, boil-off loss, time windows at the ports, or load dependent fuel consumption for the ships. With these simplifications, a mixed integer linear programming (MILP) model was formulated that can solve realistic problems within a few hours of computation time. Both strategic infrastructure decisions and tactical planning are simultaneously optimized. The multi-period formulation allows for a more detailed evaluation of the storage tank sizes and inventory, offering the possibility for a more efficient routing. The multi-period formulation is illustrated by comparison to the singleperiod solution of a case study based on the emerging LNG market in the Gulf of Bothnia.

\section{Problem description}

In this paper we present a multi-period formulation as an extension of the model presented in Bittante et al. (2016). The problem tackled is a regional supply of LNG from a set of potential supply ports to inland end customers through a set of potential satellite terminals. At the supply ports LNG is loaded to specially designed vessels and transported to the satellite terminals. LNG can also be transported by truck from the ports to inland customers. Satellite terminals have potential locations and can be activated or not according to the overall objective. Potential satellite terminals and inland customers have given fixed demands for the time horizon considered, which must be satisfied either by transported LNG or by an alternative fuel. This generic fuel is merely used to allow the model to partly or fully exclude customers from the LNG supply chain; therefore we do not consider the transportation costs of the alternative fuel. It should be noted that the demand sites represent cluster of consumers, which justifies the use of multiple fuels. The fleet of vessels can be freely chosen from a set of heterogeneous ship types, each of which has a given cruising speed, capacity, fuel consumption and loading/unloading rate. The ships can perform split delivery and no limitations on routes or ship-port connections are imposed. Restrictions regarding the maximum amount of $\mathrm{LNG}$ available at the 
supply ports is included in the formulation and can be parametrically activated. LNG tank trucks for the landbased transportation are chosen from a homogeneous fleet of given capacity and fuel consumption and cannot perform split delivery. Each truck is associated to a single supply or satellite terminal, cannot be swapped among ports and is limited to a maximum travelling distance. The number of trucks is constrained by the number of filling stations at the ports and the weekly working hours at the facilities. Inland customers are assumed to have enough storage capacity to stock the full LNG demand for the time horizon and no investment costs are considered at these sites. However, size dependent investment costs for the storage tanks are considered at the activated satellite terminals. There are three key decisions in the optimization: to locate the satellite terminals, to determine the fleet and routing for both maritime and land transport, and to size the storage tanks at the satellite terminals and to determine their inventory levels.

\section{Mathematical model}

\subsection{Sets and variables}

Let $T$ denote the set of time periods indexed by $t$. Let $J$ and $S$ denote the sets of satellite terminals and supply ports, respectively. The set of all ports is denoted by $P=$ $J \cup S$. Let $D$ denote the set of inland customers and $L=$ $J \cup D$ denote the set of all customers of given demand $D_{l, t}$. The indexed set of LNG ship types is $K$. The arc set $A$ is defined as $\{(p, m): p \in P, m \in P, p \neq m\}$ and represents all the sailing legs between pairs of ports. Let $B=\left\{(p, l): p \in P, l \in L, d_{p, l} \leq d^{\text {max }}\right\}$ denote the landbased port-consumer connection, the distance of which is below an upper limit $d^{\text {max }}$. Maritime routing is described by the use of three sets of variables: Integer variables $y_{p, m, k, t}$ indicate how many times ship type $k$ travels the sailing leg $(p, m) \in A$ in time period $t$. Continuous variables $x_{p, i, k, t}$ specify the number of LNG loads transported by ship type $k$ on the route $(p, i) \in A$ in time period $t$. The ship types composing the fleet are given by binary variables $z_{k}$. Three sets of variables are also used to model the land-based transport. Continuous variables $q_{p, l}$ express the amount of LNG transported on the route $(p, l) \in B$. The number of allocated trucks per port and the number of trips per route are given by integer variables $z_{p}$ and $z_{p, l}$, respectively. The activation of satellite terminals is controlled by the binary decision variables $w_{i}$. Continuous variables $s_{i}$ and $q_{i, t}^{S}$ denote the storage size at the activated satellite terminals and the inventory at the beginning of each time period, respectively. Finally, the supply of alternative fuel to the consumers is represented by decision variables $q_{l}^{A}$.

\subsection{Mathematical formulation}

The objective of the problem is to minimize the combined fuel, operation and investment costs. The first term in Eq. (2), the fuel cost, is given as the quantity of LNG and/or alternative fuel used multiplied by the specific fuel price. The second term, representing the operation cost, is the sum of port calls fees, costs of ship propulsion, chartering of the ships, and truck fuel consumption. Finally, the investment cost includes the truck purchase and the construction of the satellite terminal infrastructure and storages. The parameter

$$
\gamma=\frac{1}{365 \mathrm{~d}} \cdot \frac{e}{1-(1+e)^{-n}}
$$

in the last term of Eq. (2) rescales the total investment cost to the contribution for the time horizon $H$ considered in the optimization, where $e$ is the interest rate and $n$ is the life length of the investment.

The model is formulated as

$$
\begin{aligned}
& \min C^{t o t} \\
& =\sum_{s \in S} \sum_{i \in J} \sum_{k \in K} \sum_{t \in T} C_{s}^{G} Q_{k} x_{s, i, k, t} \\
& +N\left(\sum_{s \in S} \sum_{l \in L} C_{s}^{G} q_{s, l}+\sum_{l \in L} C^{A} q_{l}^{A}\right) \\
& +\sum_{(p, m) \in P} \sum_{k \in K} \sum_{t \in T} C_{p} y_{p, m, k, t} \\
& +N H \sum_{k \in K} C_{k}^{R} Z_{k} \\
& +\sum_{(p, m) \in P} \sum_{k \in K} \sum_{t \in T} C_{k}^{F} d_{p, m} y_{p, m, k, t} \\
& +2 N \sum_{p \in P} \sum_{l \in L} C^{F} d_{p, l}^{L} Z_{p, l} \\
& +\gamma N H\left[I^{T} \sum_{p \in P} z_{p}+\sum_{i \in J}\left(I^{W} w_{i}+I^{S} S_{i}\right)\right] \\
& \sum_{p \in P} \sum_{k \in K} Q_{k} x_{p, i, k, t}-\sum_{j \in J} \sum_{k \in K} Q_{k} x_{i, j, k, t} \\
& +\sum_{p \in P}^{j \in J} q_{p, i}-\sum_{l \in L} q_{i, l}+q_{i}^{A} \\
& +q_{i, t}^{S} \geq D_{i, t} H \forall i \in J, t \in T \text {. } \\
& \sum_{p \in P} q_{p, d}+q_{d}^{A} \geq D_{d, t} H \quad \forall d \in D, t \in T \\
& \begin{aligned}
\left(1-f^{S}\right) s_{i} \geq q_{i, t}^{S} & +\sum_{p \in P} \sum_{k \in K} Q_{k} x_{p, i, k, t} \\
& -\sum_{j \in J} \sum_{k \in K} Q_{k} x_{i, j, k, t} \forall i
\end{aligned}
\end{aligned}
$$




$$
\begin{gathered}
q_{i, t}^{S} \geq f^{S} s_{i} \quad \forall i \in J, t \in T \\
q_{i, t}^{S}=q_{i, t-1}^{S}+\sum_{p \in P} \sum_{k \in K} Q_{k} x_{p, i, k, t-1} \\
\quad-\sum_{j \in J} \sum_{k \in K} Q_{k} x_{i, j, k, t-1} \\
-\sum_{l \in L} q_{i, l}-D_{i, t-1} H w_{i} \quad \forall i \\
\in J, t \in T \text { and } t>1
\end{gathered}
$$$$
q_{i, t_{\text {first }}}^{S}=q_{i, t_{\text {last }}}^{S}+\sum_{p \in P} \sum_{k \in K} Q_{k} x_{p, i, k, t_{\text {last }}}
$$$$
-\sum_{j \in J} \sum_{k \in K} Q_{k} x_{i, j, k, t_{\text {last }}}
$$$$
-\sum_{l \in L} q_{i, l}
$$$$
\text { - } D_{i, t_{\text {last }}} H w_{i} \quad \forall i \in J
$$$$
\sum_{p \in P} \sum_{k \in K} \sum_{t \in T}\left(y_{p, i, k, t}+y_{i, p, k, t}\right) \leq M w_{i} \forall i \in J
$$$$
s_{i} / \mathrm{MWh} \leq M w_{i} \quad \forall i \in J
$$$$
y_{p, i, k, t} \geq x_{p, i, k, t} \forall p \in P, i \in J, k \in K, t \in T
$$

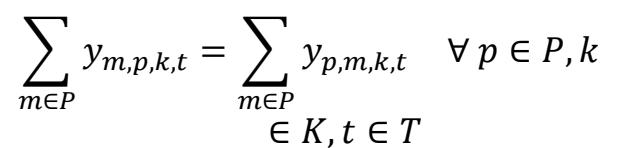$$
\sum_{p \in P} x_{p, i, k, t} \geq \sum_{p \in P} x_{i, p, k, t} \quad \forall i \in J, k \in K, t
$$$$
\in T
$$$$
M x_{p, i, k, t} \geq y_{p, i, k, t} \quad \forall p \in P, i \in J, k \in K, t
$$$$
\in T
$$

$$
\begin{aligned}
a_{k} H z_{k} \geq \frac{1}{v_{k}} \sum_{(p, m) \in P} d_{p, m} y_{p, m, k, t} \\
+\sum_{p \in P}\left(t_{\mathrm{p}}^{\mathrm{B}} \sum_{m \in P} y_{p, m, k, t}\right) \\
+\frac{2}{r_{k}} \sum_{s \in S} \sum_{p \in P} Q_{k} x_{s, p, k, t} \forall k \\
\in K, t \in T
\end{aligned}
$$

$$
\sum_{i \in J} \sum_{k \in K} Q_{k} x_{s, i, k, t}+\sum_{l \in L} q_{s, l} \leq Q_{s}^{U} H \forall s \in S, t
$$

$$
\begin{gathered}
\sum_{l \in L} q_{i, l} \leq M w_{i} \text { MWh } \quad \forall i \in J \\
z_{p, l} \geq \frac{q_{p, l}}{Q} \quad \forall p \in P, l \in L \\
z_{p} \leq Z_{p}^{U} \quad \forall p \in P \\
\sum_{l \in L} z_{p, l} \leq \frac{5}{7} H Z_{p}^{U} \quad \forall p \in P \\
a H z_{p} \geq \sum_{l \in L}\left(\frac{2}{v} d_{p, l}^{L}+t^{O}\right) z_{p, l} \quad \forall p \in P
\end{gathered}
$$

\subsection{Constraints}

Most of the constraints are identical or similar to the ones for the single period model, with the addition of the time period index. New sets of constraints are required to control the tank storage mass balance and sizing. Constraints (3) and (4) guarantee that the demand is fulfilled at the satellite terminals and at the inland consumers, respectively. Constraints (5) and (6) define the tank storage size, ensuring sufficient capacity for all the LNG delivered in the time period and for an additional fraction (heel). The storage mass balance is controlled by constraints (7), taking into account the storage levels between time periods. Rolling horizon constraints (eq. (8)) make the storage level at the beginning of the first time-period the same as that in the end of the last period. The activation of the satellite terminals is defined by constraints (9). If a terminal is activated, constraints (10) permit the existence of a storage tank by allowing variables $s_{i}$ to be positive. As in the single-period formulation, four sets of constraints (eq. (11)-(14)) control the maritime routing. Constraints (15) determine the fleet composition (in terms of ship types) based on the ship time usage. Constraints (16) limit the amount of LNG available at the supply ports. Land-based transportation is controlled by five sets of constraints. Constraints (17) ban transportation of LNG from a non-activated satellite terminal. The number of required truck voyages from port to customer is obtained from constraints (18). The total number of trucks and truck voyages from each port are limited based on the maximum number of truck loads per day, by constraints (19) and (20). Finally, constraints (21) guarantee a sufficient number of trucks per port to carry out the total land-based LNG delivery.

\section{Case study}

The presented model has been applied to a study of LNG delivery in the Gulf of Bothnia (i.e., the northern part of the Baltic Sea). This fictitious was created inspired by 
several LNG-related projects approved or under discussion in this region.

Two supply terminals (Tornio, Stockholm), one fixed satellite terminal (Pori, 30,000 $\mathrm{m}^{3}$ ) and three potential ones (Turku, Vaasa, Umeå) on the coasts of Finland and Sweden have been preselected. A total of twenty clusters distributed in Finland and Sweden were identified as inland customers. Demands were assigned as gross estimates based on population, extent of industrial activity and time horizon considered. Five ship types, with capacities $Q_{k}$ of $3,000 \mathrm{~m}^{3}, 5,000 \mathrm{~m}^{3}$, $6,500 \mathrm{~m}^{3}, 7,500 \mathrm{~m}^{3}$ and $10,000 \mathrm{~m}^{3}$ were selected, with parameters inspired by small-scale LNG carrier designs by Wärtsilä (Wärtsilä 2015). Maritime distances were obtained from an online tool for calculation of distances between sea ports (Sea-Distances.org 2015), while road distances were collected from a web mapping service (Google Maps). A maximum distance $d^{\max }=350 \mathrm{~km}$ was used to identify the feasible port-customer road connections. The availability of vessels and trucks is a portion of the total time horizon, allowing for some extra time. An availability of $95 \%$ was used for the ships $\left(a_{k}=0.95\right)$ while the corresponding number for trucks was $a=0.298$; the latter factor also considers a rescaling of the total time to ten-hour working days in a five-day working week. The optimization was first performed for a 10-day single-period time horizon $(H)$ and then successively for three identical time periods $(N=3)$ of 10 days each to study the impact of the multi-period formulation on the overall optimization. Tables with the numerical values of all the parameters used in the model are presented in the Appendix.

The MILP model was implemented in AIMMS 4.8 using the IBM ILOG CPLEX Optimizer (AIMMSCPLEX). The problem of the case study results in 609 integer and 444 continuous variables. The solution time of this size of problem varied between $30 \mathrm{~s}$ and $40 \mathrm{~min}$ depending on parameter values, on a computer with a 3.5 GHz Intel Core i7 processor and $32 \mathrm{~GB}$ of RAM.

\subsection{Single-period results}

We first present results for the single-period case, where the price of LNG at the two supply ports is identical, $\mathrm{C}_{s}^{G}=30 € / \mathrm{MWh}$, and the price of alternative fuel at the consumers is $C^{A}=40 € / \mathrm{MWh}$. Figure 1 illustrates the optimal maritime routing (curved arrowed arcs) and port-to-customer truck connections (straight arrowed lines). Detailed numerical results of the optimization are reported in Tables 1 and 2.

The results show that all the customers are served partially or entirely with LNG. Only two customers are partially supplied with alternative fuel (Sollefteå and Kokkola, both with $0.038 \mathrm{GWh}$ ). One ship of Type 3 $\left(6,500 \mathrm{~m}^{3}\right)$ is needed for the LNG maritime distribution to the satellite terminals, which in addition to the one in Pori have been activated in Umeå and Vaasa. The storage sizes of the latter ones are about $7500 \mathrm{~m}^{3}$ and
$2500 \mathrm{~m}^{3}$, respectively, while Pori has a fixed capacity of $30,000 \mathrm{~m}^{3}$. The maritime routing is reported in Table 1 . One split delivery is performed between Umeå, Pori and Vaasa. Totally, 113.4 GWh of LNG is shipped to the three satellite terminals; one third from Stockholm and the remaining from Tornio. The total amount of LNG transported by truck is $113 \mathrm{GWh}$, in 364 trips. The number of trucks per port is indicated in Table 2, ranging from 1 in Vaasa to 17 in Tornio. Supply ports have the highest number as they serve the majority of the land customers reached. Among the satellite terminals, Pori has the largest amount of LNG for truck transport, and therefore a high number of allocated trucks. For this case, the overall optimal cost is 32.406 $€ / M W h$.

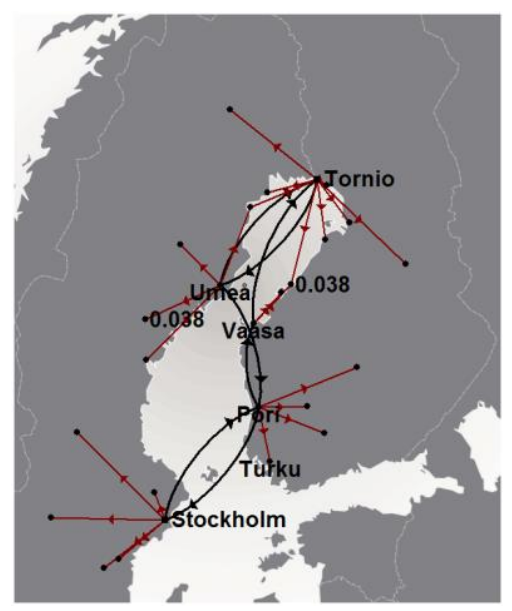

Figure 1. Optimal satellite terminal locations and LNG distribution from ports for the single-period case. Straight arrows indicate land transport by truck and arrowed arcs maritime routing. Alternative fuel is reported in GWh.

Table 1. Optimal results for the maritime routing for the single-period case, where $y$ denotes the number of trips and $x$ the ship loads.

\begin{tabular}{lcc}
\hline Route & $y_{p, m, k}$ & $x_{p, i, k}$ \\
\hline Stockholm-Pori & 1 & 0.99 \\
Pori-Stockholm & 1 & - \\
Tornio-Umeå & 2 & 2.00 \\
Umeå-Tornio & 1 & - \\
Umeå-Pori & 1 & 0.97 \\
Pori-Vaasa & 1 & 0.35 \\
Vaasa-Tornio & 1 & - \\
\hline
\end{tabular}

Table 2. Number of trucks per port and amount of LNG delivered to customers by truck.

\begin{tabular}{lcc}
\hline Port & $z_{p},-$ & LNG trucked, GWh \\
\hline Tornio & 17 & 42.5 \\
Stockholm & 15 & 37 \\
Pori & 8 & 21 \\
Umeå & 4 & 9.1 \\
Vaasa & 1 & 3.4 \\
\hline
\end{tabular}




\subsection{Multi-period results}

For the multi-period case, we consider three identical periods with a rolling time horizon. As it is interesting to optimize the storage tank sizes in combination with the maritime routing, the land-based transport solution was taken to be independent of the time period, so the amount of LNG delivered by truck, the number of trucks and the related port-customers connections are identical in the three periods. However, they need not be identical to the solution of the single-period case, as the landbased transport will still be optimized together with the time-period-dependent maritime routing and storage.

Results are presented in Figure 2 and Tables 3-4. The same satellite terminals as in the single-period case are seen to be activated, but the storage tank sizes are about 4,000 $\mathrm{m}^{3}$ larger: Umeå and Vaasa now have tanks of about $11,500 \mathrm{~m}^{3}$ and $6,500 \mathrm{~m}^{3}$, respectively. This increase in investment cost is outweighed by a shift to a smaller ship of Type 2, which is sufficient for carrying out the maritime LNG deliveries. Figure 2 a-c presents the routing, which is seen to be different for the time periods. The total amount of LNG transported by ship in all three time periods is $341.8 \mathrm{GWh}$, supplied roughly equally from Stockholm and Tornio. The amount of LNG transported by trucks is about $337.5 \mathrm{GWh}$. Comparing these numbers with the results for the singleperiod problem, on average and per period, LNG deliveries by sea have increased by $0.5 \mathrm{GWh}$ while deliveries by road have decrease by $0.5 \mathrm{GWh}$. In more detail, the LNG transported by truck from Tornio and Pori has decreased by $1 \mathrm{GWh}$ and $3.2 \mathrm{GWh}$, respectively, while the land transport from Vaasa has increased by $3.7 \mathrm{GWh}$. This explains how the increase in maritime delivery has been used to partially outweigh the decrease in Tornio by redistributing the land-based deliveries between Pori and Vaasa (see Table 4). The remaining reduction in LNG use found in the optimal solution of the multi-period case has been fulfilled by a larger quantities of alternative fuel (see Figure 2, where alternative fuel amounts reported in GWh).

Table 3. Optimal maritime routing for multi-period case

\begin{tabular}{lccc}
\hline Route & Time period & $y_{p, m, k, t}$ & $x_{p, i, k, t}$ \\
\hline Stockholm-Pori & 1 & 1 & 1 \\
Pori-Stockholm & 1 & 1 & - \\
Tornio-Umeå & 1 & 2 & 2.00 \\
Umeå-Tornio & 1 & 3 & - \\
Tornio-Vaasa & 1 & 1 & 0.84 \\
Vaasa-Umeå & 1 & 1 & 0.09 \\
Stockholm-Pori & 2 & 3 & 2.95 \\
Pori-Stockholm & 2 & 3 & - \\
Tornio-Umeå & 2 & 1 & 0.93 \\
Umeå-Tornio & 2 & 1 & - \\
Stockholm-Pori & 3 & 2 & 2.00 \\
Pori-Stockholm & 3 & 2 & - \\
Tornio-Umeå & 3 & 1 & 1.00 \\
Umeå-Tornio & 3 & 1 & - \\
Tornio-Vaasa & 3 & 1 & 1.00 \\
Vaasa-Tornio & 3 & 1 & - \\
\hline
\end{tabular}

Table 4. Number of trucks per port and amount of LNG delivered to customers by truck.

\begin{tabular}{lcc}
\hline Port & $z_{p},-$ & LNG trucked, GWh \\
\hline Tornio & 16 & 41.5 \\
Stockholm & 15 & 37 \\
Pori & 6 & 17.8 \\
Umeå & 4 & 9.1 \\
Vaasa & 3 & 7.1 \\
\hline
\end{tabular}

Figure 3 and 4 show the use of the storage tank capacity by reporting the data of the stored and unloaded LNG at each port per time period. The stored LNG refer
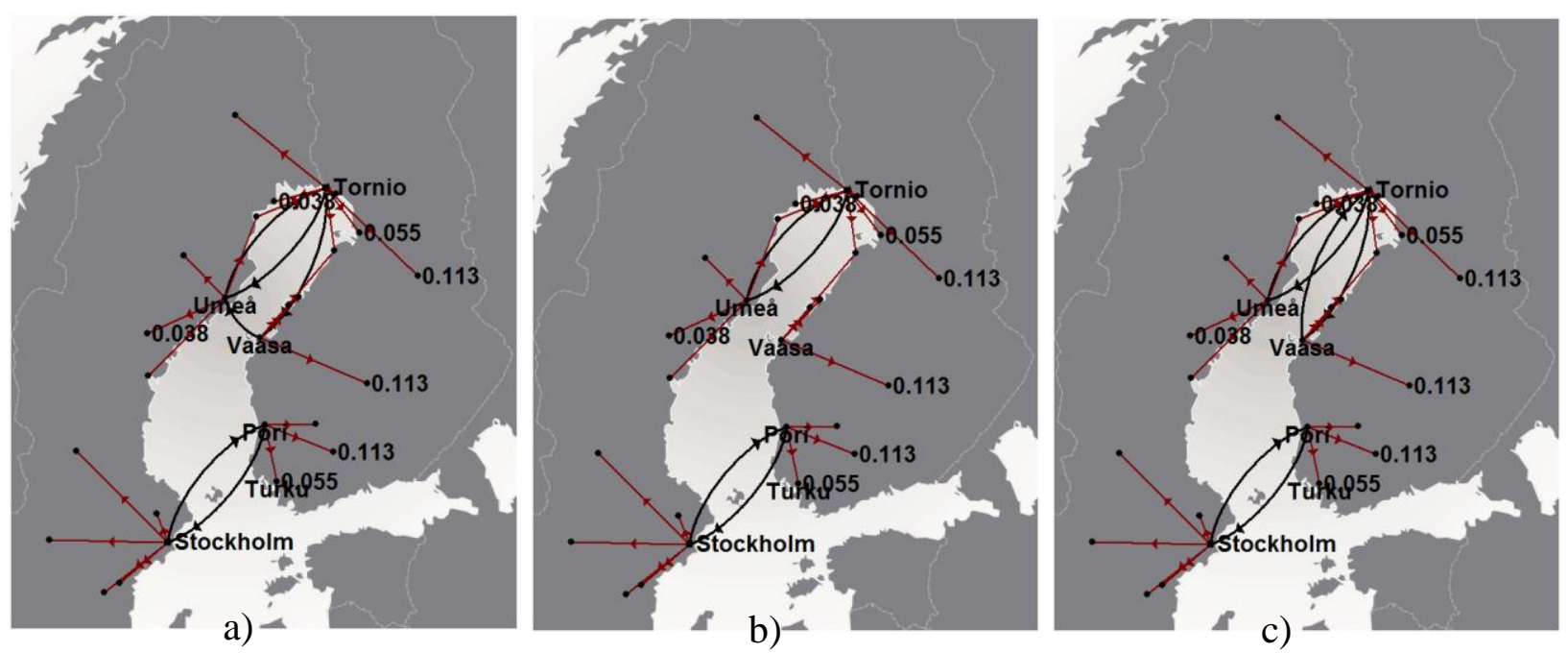

Figure 2. Optimal satellite terminal locations and LNG distribution from supply to satellite ports for the multi-period case. a) Time period 1, b) Time period 2, and c) Time period 3. 
to the state at the beginning of the time periods, while the unloaded quantities refer to the ship deliveries for the time period. It can be seen that a bigger inventory is used for periods when the unloaded LNG is insufficient to cover the demand. This is particular the case for Vaasa, which does not have any ship visits during period 2 (Figure 4 vs. Figure 2 b) and instead uses the storage to satisfy the local demand and the demands of its inland customers (Figure 3). The overall cost for the multiperiod solution is $32.333 € / \mathrm{MWh}$, which is more than 7 cents lower than the costs of the single-period solution, yielding a yearly saving of about half a million euro.

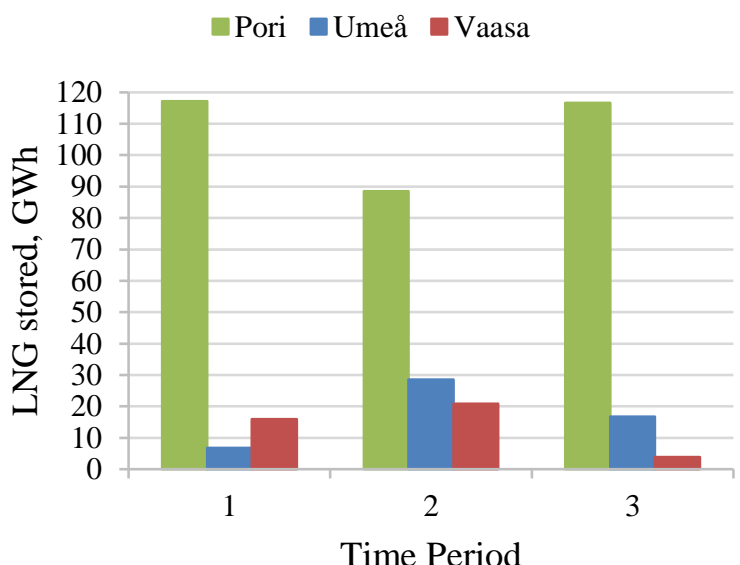

Figure 3. Amount of LNG stored at the satellite terminals in the beginning of every time period.

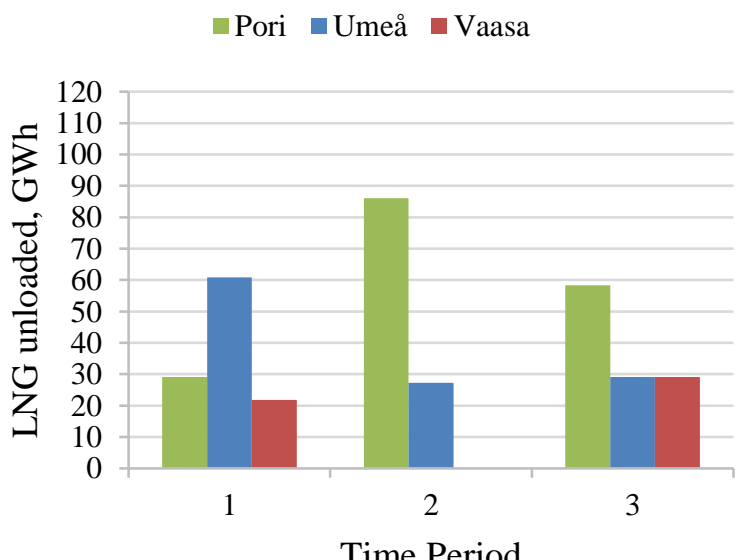

Figure 4. Amount of LNG unloaded at the satellite terminals per time period.

\section{Conclusions and future work}

An MILP model for the optimal design of a small-scale LNG supply chain has been presented. The model addresses both strategic and tactical aspects, often treated separately in logistics studies. This is realized by a simultaneous minimization of investment and operation costs in the objective function. The extension to a multi-period formulation makes it possible to optimize storage tank sizing and inventory. The optimal solution gives the location of the satellite terminals (to be built), the fleet configuration in terms of number and type of ships and number of tank trucks per port, the associated distribution network (maritime routing and road connections), the size of the storage tanks at the satellite terminals, and their inventory levels. The results from a case study demonstrate the advantages of a multiperiod formulation on the overall optimal supply chain design. In small-scale designs where demands and distances are relatively small, it is not clear at the outset whether, in terms of costs, operations should drive investments decisions or vice versa. This can be clarified by using the proposed model, which has proven to be a valuable tool for early evaluation of new LNG projects, but also for initial planning and design. The simplicity and flexibility of the formulation make it an ideal basis for studies of other supply chains, e.g., in the evaluation and upgrading of existing networks.

In the future work, attention will be directed to consider uncertainties since transportation conditions, equipment availability and market circumstances may vary. A stochastic model could be developed to consider parameters variation and different scenarios to find solutions that are robust, i.e., insensitive to parametric changes.

\section{Acknowledgements}

This work was carried out in the Efficient Energy Use (EFEU) research program coordinated by CLIC Innovation Ltd. with funding from the Finnish Funding Agency for Technology and Innovation (Tekes) and participating companies. The financial support is gratefully acknowledged.

\section{Appendix}

In this appendix we report all the numerical values of the parameters of the mathematical model. Table A.1 reports the sea distances expressed in kilometers. Parameters regarding port specifications are given in Table A.2. The parameter $Z_{p}^{U}$ limiting the truck trips has been estimated considering the number of loading stations available at the ports ( 5 for supply ports, 3 for satellite terminals), an average two-hour time for loading operations and a ten-hour service at the port for twenty working days a month. Table A.3 reports parameters of the different ship types. Other miscellaneous model parameters are listed in Table A.4. Finally, Table A.5 reports the road distances between ports and customers, as well as the customers' demands. 
Table A. 1. Sea distances between ports (Sea-Distances.org 2015).

\begin{tabular}{|c|c|c|c|c|c|c|}
\hline$d_{p, m}, k m$ & Tornio & Stockholm & Turku & Pori & Vaasa & Umeå \\
\hline Tornio & 0 & 809 & 885 & 559 & 373 & 338 \\
\hline Stockholm & 809 & 0 & 324 & 422 & 580 & 632 \\
\hline Turku & 885 & 324 & 0 & 315 & 485 & 452 \\
\hline Pori & 559 & 422 & 315 & 0 & 253 & 288 \\
\hline Vaasa & 373 & 580 & 485 & 253 & 0 & 115 \\
\hline Umeå & 338 & 632 & 452 & 288 & 115 & 0 \\
\hline
\end{tabular}

Table A. 2. Port specific parameters

\begin{tabular}{llllll}
\hline Ports & $C_{p}, €$ & $C_{s}^{G}, € / M W h$ & $Q_{s}^{U}, G W h / d$ & $t_{p}^{B}, h$ & $Z_{p}^{U},-$ \\
\hline Tornio & 5,000 & 30 & 40 & 5 & 25 \\
Stockholm & 5,000 & 30 & 40 & 5 & 25 \\
Turku & & & 5 & 15 \\
Pori & & & 5 & 15 \\
Vaasa & & & 5 & 15 \\
Umeå & & & 5 & 15 \\
\hline
\end{tabular}

Table A. 3. Ship-related parameters

\begin{tabular}{lllllll}
\hline Ship Type & $a_{k},-$ & $C_{k}^{F}, € / \mathrm{km}$ & $C_{k}^{R}, € / d$ & $Q_{k}, M W h(\mathrm{m3})$ & $r_{k}, M W(\mathrm{~m} 3 / \mathrm{h})$ & $v_{k}, \mathrm{~km} / \mathrm{h}$ \\
\hline Type 1 & 0.95 & 4 & 11,000 & $17,499(3,000)$ & $4666.4(800)$ & 21 \\
Type 2 & 0.95 & 5 & 14,000 & $29,165(5,000)$ & $4666.4(800)$ & 23 \\
Type 3 & 0.95 & 5 & 17,000 & $37,914.5(6,500)$ & $4666.4(800)$ & 24 \\
Type 4 & 0.95 & 6 & 19,000 & $43,747.5(7,500)$ & $4666.4(800)$ & 25 \\
Type 5 & 0.95 & 6 & 23,000 & $58,333.0(10,000)$ & $4666.4(800)$ & 26 \\
\hline
\end{tabular}

Table A. 4. Other model parameters

\begin{tabular}{ll}
\hline Parameter & \\
\hline$a,-$ & 0.298 \\
$C^{A}, € / M W h$ & 40 \\
$C^{F}, € / k m$ & 1 \\
$e,-$ & 0,01 \\
$f^{S},-$ & 0.1 \\
$H, d$ & 10 \\
$I^{T}, €$ & $2,000,000$ \\
$I^{S}, € / M W h$ & 200 \\
$I^{W}, €$ & $20,000,000$ \\
$n, a$ & 30 \\
$N,-$ & 3 \\
$Q, M W h\left(m^{3}\right)$ & $320.8(55)$ \\
$t^{O}, h$ & 2 \\
$v, k m / h$ & 50 \\
$\gamma, 1 / d$ & 0.0001 \\
\hline
\end{tabular}


Table A. 5. Road distances between ports and customers (Google Maps) and customer daily demands.

$d_{p, l}^{L}, k m$

\begin{tabular}{|c|c|c|c|c|c|c|c|c|c|c|c|c|c|c|c|c|c|c|c|c|c|c|c|c|}
\hline & $\stackrel{\overrightarrow{3}}{=}$ & 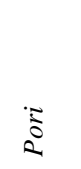 & $\begin{array}{l}\tilde{\Xi} \\
\vdots \\
\vdots\end{array}$ & 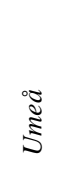 & $\stackrel{\Xi}{\Xi}$ & $\frac{\stackrel{\Xi}{\sigma}}{\frac{\pi}{2}}$ & 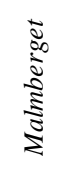 & 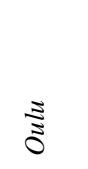 & 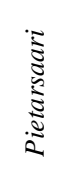 & $\stackrel{\mathscr{\Xi}}{\widetilde{\Xi}}$ & 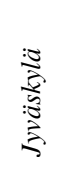 & 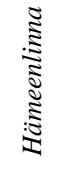 & 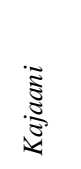 & 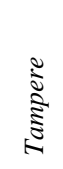 & 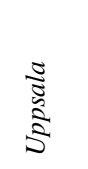 & 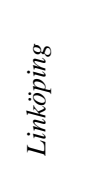 & 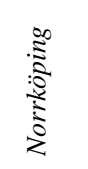 & 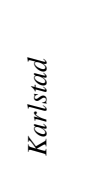 & $\stackrel{5}{2}$ & 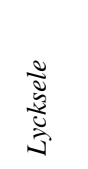 & $\begin{array}{l}\stackrel{\Xi}{\Xi} \\
\stackrel{\Xi}{\Xi} \\
n\end{array}$ & छัँ & $\stackrel{\Xi}{\Xi}$ & 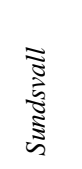 \\
\hline Tornio & 778 & 639 & 450 & 386 & 28 & 330 & 260 & 131 & 368 & 175 & 470 & 658 & 313 & 618 & 957 & 1,219 & 1,182 & 1,198 & 945 & 388 & 589 & 206 & 130 & 649 \\
\hline Stockholm & 1,000 & 455 & 747 & 638 & 1050 & 868 & 1,138 & 1,153 & 845 & 850 & 622 & 456 & 1,335 & 476 & 70 & 197 & 160 & 305 & 307 & 717 & 493 & 1228 & 906 & 375 \\
\hline Turku & 0 & 142 & 334 & 1,163 & 752 & 436 & 1,036 & 647 & 413 & 951 & 308 & 143 & 622 & 162 & 1,000 & 511 & 474 & 599 & 590 & 581 & 647 & 563 & 907 & 1,425 \\
\hline Pori & 142 & 0 & 191 & 1,024 & 612 & 309 & 897 & 508 & 286 & 813 & 263 & 186 & 577 & 111 & 459 & 654 & 617 & 742 & 864 & 438 & 508 & 434 & 767 & 1,285 \\
\hline Vaasa & 334 & 191 & 0 & 835 & 424 & 121 & 709 & 320 & 98 & 624 & 267 & 321 & 367 & 240 & 686 & 948 & 911 & 927 & 674 & 1,000 & 1,000 & 246 & 579 & 1,097 \\
\hline Umeå & 1,163 & 1,024 & 835 & 0 & 413 & 1,000 & 501 & 516 & 752 & 213 & 1,000 & 441 & 698 & 360 & 572 & 833 & 797 & 812 & 560 & 128 & 203 & 591 & 265 & 264 \\
\hline
\end{tabular}

$D_{l, t}, G W h / d$

$\begin{array}{llllllllllllllllllllllllllllllll}1 & 4 & 1 & 3 & 0.7 & 0.1 & 0.6 & 1 & 0.3 & 0.3 & 0.3 & 0.3 & 0.3 & 0.5 & 1 & 1 & 1 & 0.5 & 0.2 & 0.5 & 0.1 & 1.2 & 0.1 & 0.3\end{array}$




\section{Nomenclature}

Sets

$J \quad$ Set of satellite terminals

$D \quad$ Set of inland customers

K Set of ship types

$L \quad$ Set of customers

$P \quad$ Set of ports

$S \quad$ Set of supply ports

$T \quad$ Set of time periods

Indices

$j \quad$ Satellite terminals $j \epsilon I$

$d \quad$ Inland customers $d \epsilon D$

$k \quad$ Ship types $k \epsilon K$

$l \quad$ Customers $l \in L$

$p, m \quad$ Ports $(p, m) \epsilon P$

$s \quad$ Supply ports $s \in S$

Variables

$q_{l}^{A} \quad$ Continuous variable indicating the amount of energy from alternative fuel, MWh

$q_{p, l} \quad$ Continuous variable indicating the amount of energy from LNG trucked, MWh

$s_{i} \quad$ Continuous variable indicating the size of the tank storage, MWh

$z_{p} \quad$ Integer variable indicating number of trucks per port, -

$z_{p, l} \quad$ Integer variable indicating number of truck trips between $p$ and $l$,-

$w_{i} \quad$ Binary variable, $w_{i}=1$ if satellite terminal $i$ is activated, -

$x_{p, i, k, t}$ Continuous variable indicating ship load transported, -

$y_{p, m, k, t}$ Integer variable indicating number of time the route between $p$ and $m$ is travelled, -

$z_{k} \quad$ Binary variable indicating the ship types, -

$q_{i, t}^{S} \quad$ Continuous variable indicating amount of LNG stored at the beginning of the time period, MWh

\section{Parameters}

a Truck availability, -

$a_{k} \quad$ Ship availability, -

$C^{A} \quad$ Price of the alternative fuel, $€ / \mathrm{MWh}$

$C_{s}^{G} \quad$ Price of LNG in supply port $s, € / \mathrm{MWh}$

$C_{p} \quad$ Port call cost, $€$

$C^{F} \quad$ Truck fuel consumption cost, $€ / \mathrm{km}$

$C_{k}^{F} \quad$ Ship propulsion cost, $€ / \mathrm{km}$

$C_{k}^{R} \quad$ Ship renting cost, $€ / \mathrm{d}$

$d_{p, m} \quad$ Maritime distance, $\mathrm{km}$

$d_{p, l}^{L} \quad$ Road distance, $\mathrm{km}$
$D_{l, t} \quad$ Energy demand, MWh/d

$e \quad$ Interest rate, -

$f^{\mathrm{S}} \quad$ Fraction of storage capacity for LNG heel, -

$H \quad$ Time horizon, $\mathrm{d}$

$I^{T} \quad$ Truck investment cost, $€$

$I^{S} \quad$ Tank storage investment cost, $€ / \mathrm{MWh}$

$I^{W} \quad$ Fixed investment cost of satellite terminal, $€$

$M \quad$ Big-M parameter, -

$n$ Payback time, year

$N \quad$ Number of time periods, -

$Q \quad$ Truck capacity, MWh

$Q_{S}^{U} \quad$ Maximum amount of LNG available at the supply port, $\mathrm{MWh} / \mathrm{d}$

$Q_{k} \quad$ Ship capacity, MWh

$r_{k} \quad$ Loading/unloading rate, MW

$t_{p}^{B} \quad$ Berthing time, $\mathrm{h}$

$t^{O} \quad$ Operation time loading trucks, $\mathrm{h}$

$Z_{p}^{U} \quad$ Maximum number of truck's loads per day in port $p, 1 / \mathrm{d}$

$v \quad$ Truck average speed, $\mathrm{km} / \mathrm{h}$

$v_{k} \quad$ Ship average cruising speed, $\mathrm{km} / \mathrm{h}$

$\gamma \quad$ Investment instalment factor, $1 / \mathrm{d}$

\section{References}

AIMMS-CPLEX, AIMMS-CPLEX. Available at: https://aimms.com/english/developers/resources/sol vers/cplex [Accessed June 2, 2017].

Al-Khayyal, F. \& Hwang, S.-J., 2007. Inventory constrained maritime routing and scheduling for multi-commodity liquid bulk, Part I : Applications and model. European Journal of Operational Research, 176(1), pp.106-130. Available at: http://dx.doi.org/10.1016/j.ejor.2005.06.047.

Andersson, H. et al., 2010. Computers \& Operations Research Industrial aspects and literature survey: Combined inventory management and routing. Computers and Operation Research, 37(9), pp.1515-1536. Available at: http://dx.doi.org/10.1016/j.cor.2009.11.009.

Baldacci, R., Battarra, M. \& Vigo, D., 2009. Valid inequalities for the fleet size and mix vehicle routing problem with fixed costs. Networks, 54(4), pp.178-189. Available at: http://doi.wiley.com/10.1002/net.20331.

Bittante, A. et al., 2016. Mixed integer optimization of an LNG supply chain in the Baltic Sea region. In The 29th International Conference on Efficiency, Cost, Optimization, Simulation and Environmental Impact of Energy Systems. p. P624.

Braekers, K., Ramaekers, K. \& Nieuwenhuyse, I. Van, 2015. The Vehicle Routing Problem: State of the Art Classification and Review. Computers \& 
Industrial Engineering. Available at:

http://www.sciencedirect.com/science/article/pii/S0 360835215004775 [Accessed June 2, 2017].

Drexl, M. \& Schneider, M., 2015. A survey of variants and extensions of the location-routing problem.

European Journal of Operational Research, 241(2), pp.283-308. Available at:

http://www.sciencedirect.com/science/article/pii/S0 377221714006651 [Accessed June 2, 2017].

Gonzalez-Feliu, J., 2009. The N-echelon Location routing problem: concepts and methods for tactical and operational planning, Available at: https://halshs.archives-ouvertes.fr/halshs00422492/file/Multi-echelon_LRP.pdf [Accessed June 2, 2017].

Google Maps, Google Maps. Available at: https://www.google.fi/maps? source=tldsi\&hl=en [Accessed June 2, 2017].

Hoff, A. et al., 2010. Industrial aspects and literature survey: Fleet composition and routing. Computers and Operations Research, 37(12), pp.2041-2061. Available at: http://dx.doi.org/10.1016/j.cor.2010.03.015.

Koza, D.F., Ropke, S. \& Molas, A.B., 2017. The liquefied natural gas infrastructure and tanker fleet sizing problem. Transportation Research Part E, 99, pp.96-114. Available at: http://dx.doi.org/10.1016/j.tre.2017.01.003.

Sea-Distances.org, 2015. SEA-DISTANCES.ORG Distances. Available at: http://www.seadistances.org/ [Accessed June 2, 2017].

Wärtsilä, 2015. Gas Carriers. Available at: http://www.wartsila.com/products/marine-oilgas/ship-design/merchant/gas-carriers [Accessed June 2, 2017]. 